\title{
The role of replication-invariance: two answers concerning the problem of fair division when preferences are single-peaked
}

Citation for published version (APA):

Klaus, B. E. (2007). The role of replication-invariance: two answers concerning the problem of fair division when preferences are single-peaked. METEOR, Maastricht University School of Business and Economics. METEOR Research Memorandum No. 029 https://doi.org/10.26481/umamet.2007029

Document status and date:

Published: 01/01/2007

DOI:

10.26481/umamet.2007029

Document Version:

Publisher's PDF, also known as Version of record

Please check the document version of this publication:

- A submitted manuscript is the version of the article upon submission and before peer-review. There can be important differences between the submitted version and the official published version of record.

People interested in the research are advised to contact the author for the final version of the publication, or visit the DOI to the publisher's website.

- The final author version and the galley proof are versions of the publication after peer review.

- The final published version features the final layout of the paper including the volume, issue and page numbers.

Link to publication

\footnotetext{
General rights rights.

- You may freely distribute the URL identifying the publication in the public portal. please follow below link for the End User Agreement:

www.umlib.nl/taverne-license

Take down policy

If you believe that this document breaches copyright please contact us at:

repository@maastrichtuniversity.nl

providing details and we will investigate your claim.
}

Copyright and moral rights for the publications made accessible in the public portal are retained by the authors and/or other copyright owners and it is a condition of accessing publications that users recognise and abide by the legal requirements associated with these

- Users may download and print one copy of any publication from the public portal for the purpose of private study or research.

- You may not further distribute the material or use it for any profit-making activity or commercial gain

If the publication is distributed under the terms of Article $25 \mathrm{fa}$ of the Dutch Copyright Act, indicated by the "Taverne" license above, 


\section{Bettina Klaus}

The Role of Replication-Invariance: Two Answers Concerning the Problem of Fair Division when Preferences are Single-Peaked

$\mathrm{RM} / 07 / 029$

JEL code: D63, D71

\section{METE@R}

Maastricht research school of Economics of TEchnology and ORganizations

Universiteit Maastricht

Faculty of Economics and Business Administration P.O. Box 616

NL - 6200 MD Maastricht

phone : ++31433883830

fax : $\quad++31433884873$ 



\title{
The Role of Replication-Invariance: Two Answers Concerning the Problem of Fair Division when Preferences are Single-Peaked*
}

\author{
Bettina Klaus ${ }^{\dagger}$
}

July 2007

\begin{abstract}
We consider the problem of allocating an infinitely divisible commodity among a group of agents with single-peaked preferences. A rule that has played a central role in the previous analysis of the problem is the so-called uniform rule. Thomson (1995a) proved that the uniform rule is the only rule satisfying Pareto optimality, no-envy, one-sided populationmonotonicity, and replication-invariance. Replacing one-sided population-monotonicity by one-sided replacement-domination yields another characterization of the uniform rule (Thomson, 1997a). Until now, the independence of replication-invariance from the other properties in these characterizations was an open problem. In this note we prove this independence by means of a single example.

Keywords: Fair allocation, single-peaked preferences, population-monotonicity, replacement-domination, replication-invariance.

JEL classification: D63, D71.
\end{abstract}

\section{Introduction}

We consider the division of some perfectly divisible commodity among a group of agents with single-peaked preferences. This means that each agent has a most preferred amount below which and above which his welfare is decreasing. A typical example is rationing in a two-good exchange economy when prices are in disequilibrium (e.g., Benassy, 1982): if the preferences of the agents over the two-dimensional space of bundles are strictly convex, then the restrictions of these preferences to the budget lines are single-peaked. In this context Benassy (1982) considered the uniform rationing scheme. For the more general class of division problems with single-peaked preferences, this solution is known as the uniform rule. Sprumont (1991) initiated the axiomatic analysis of this class of problems and proved that the uniform rule is the only rule that satisfies Pareto optimality, no-envy, and strategy-proofness. Since then a wide literature has been concerned with the search for and analysis of rules with appealing properties. We refer the interested reader to a survey of fair allocation when preferences are single-peaked by Thomson (1997b).

\footnotetext{
*The first version of this note was written while the author was visiting the University of Rochester in 1997. I acknowledge the hospitality of the Department of Economics at the University of Rochester and wish to thank William Thomson for many helpful comments. Furthermore, I thank the Netherlands Organisation for Scientific Research (NWO) for its support under grant VIDI-452-06-013.

${ }^{\dagger}$ Department of Economics, Maastricht University, P.O. Box 616, 6200 MD Maastricht, The Netherlands; e-mail: B.Klaus@algec.unimaas.nl
} 
In this note we answer some questions asked by William Thomson (1995a,1997a). Thomson (1995a) proves that the uniform rule is the only rule satisfying Pareto optimality, noenvy, replication-invariance, and one-sided population-monotonicity. Replacing one-sided population-monotonicity by one-sided replacement-domination yields another characterization of the uniform rule (Thomson, 1997a). Until now, the independence of replicationinvariance from the other properties in the characterizations mentioned above was an open problem. In this note we prove this independence by means of a single rule that satisfies the properties named in the characterizations but not replication-invariance.

\section{The Model}

In this section we introduce the problem of fair division when preferences are single-peaked and the properties for rules that will play a central role in this paper.

There is an infinite population of potential agents, indexed by the natural numbers $\mathbb{N}$. Each agent $i \in \mathbb{N}$ is equipped with a continuous and single-peaked preference relation $R_{i}$ defined over the non-negative real numbers $\mathbb{R}_{+}$. Single-peakedness of $R_{i}$ means that there exists a point $p\left(R_{i}\right) \in \mathbb{R}_{+}$, called agent $i$ 's peak amount, with the following property: for all $x, y \in \mathbb{R}_{+}$with $x<y \leq p\left(R_{i}\right)$ or $x>y \geq p\left(R_{i}\right)$, we have $y P_{i} x .^{1}$ Each preference relation $R_{i}$ can be described in terms of the indifference function $r_{i}: \mathbb{R}_{+} \rightarrow \mathbb{R}_{+} \cup\{\infty\}$ that is defined as follows. If $x \leq p\left(R_{i}\right)$, then $r_{i}(x) \geq p\left(R_{i}\right)$ and either $r_{i}(x) I_{i} x$ (if such a point exists) or $r_{i}(x)=\infty$. If $x \geq p\left(R_{i}\right)$, then $r_{i}(x) \leq p\left(R_{i}\right)$ and $r_{i}(x) I_{i} x$ (if such a point exists) or $r_{i}(x)=0$.

By $\mathcal{R}$ we denote the class of all continuous, single-peaked preference relations over $\mathbb{R}_{+}$ and by $\mathcal{R}_{b} \subsetneq \mathcal{R}$ the subclass of preferences $R_{i} \in \mathcal{R}_{b}$ such that the corresponding indifference function $r_{i}$ is bounded, i.e., $r_{i}(0)<\infty$. By $\mathcal{N}$ we denote the class of non-empty and finite subsets of $\mathbb{N}$. For $N \in \mathcal{N}, \mathcal{R}^{N}$ denotes the set of (preference) profiles $R=\left(R_{i}\right)_{i \in N}$ such that for all $i \in N, R_{i} \in \mathcal{R} ; \mathcal{R}_{b}^{N}$ has a similar meaning.

Now, an economy can be formalized as follows. Let $\Omega \in \mathbb{R}_{+}$be the amount of an infinitely divisible commodity, the (social) endowment, that has to be distributed among a group of agents $N \in \mathcal{N}$ with profile $R \in \mathcal{R}^{N}$. ${ }^{2}$ We call a pair $e=(R, \Omega) \in \mathcal{R}^{N} \times \mathbb{R}_{+}$an economy. Let $\mathcal{E}^{N}=\mathcal{R}^{N} \times \mathbb{R}_{+}$be the class of all economies for $N \in \mathcal{N}$. Similarly, let $\mathcal{E}_{b}^{N}=\mathcal{R}_{b}^{N} \times \mathbb{R}_{+}$. A feasible allocation for $e=(R, \Omega) \in \mathcal{E}^{N}$ is a vector $x \in \mathbb{R}_{+}^{N}$ such that $\sum_{N} x_{i}=\Omega$. A (allocation) rule is a function $\varphi$ that assigns to every $N \in \mathcal{N}$ and every $e \in \mathcal{E}^{N}$ a feasible allocation, denoted $\varphi(e)$. Given $i \in N$, we call $\varphi_{i}(e)$ the allotment of agent $i$.

A standard requirement is Pareto optimality: an allocation assigned by the rule cannot be changed in such a way that no agent is worse off and some agent is better off.

Pareto optimality: For all $N \in \mathcal{N}$ and all $e \in \mathcal{E}^{N}$, there is no feasible allocation $x \in \mathbb{R}_{+}^{N}$ such that for all $i \in N, x_{i} R_{i} \varphi_{i}(e)$ and for some $j \in N, x_{j} P_{j} \varphi_{j}(e)$.

By the single-peakedness of the preferences it is easy to show that a rule is Pareto optimal if and only if it is same-sided, that is: for all $N \in \mathcal{N}$ and all $e \in \mathcal{E}^{N}$, either [for all $i \in N$, $\varphi_{i}(e) \leq p\left(R_{i}\right)$ ] or [for all $\left.i \in N, \varphi_{i}(e) \geq p\left(R_{i}\right)\right]$. Sprumont (1991) uses same-sidedness as definition of Pareto optimality.

\footnotetext{
${ }^{1} P_{i}$ denotes the strict preference relation associated with $R_{i}$ and $I_{i}$ the indifference relation.

${ }^{2}$ Note that free disposal of the commodity is not allowed.
} 
The following well-known property of no-envy states that no agent strictly prefers the allotment of another agent to his own allotment.

No-envy: $\quad$ For all $N \in \mathcal{N}$, all $e \in \mathcal{E}^{N}$, and all $i, j \in N, \varphi_{i}(e) R_{i} \varphi_{j}(e)$.

Next, we discuss so-called "solidarity properties" that describe the effect of certain changes in a single parameter of the economy while the other parameters are kept fixed, e.g., the population or the preferences.

Thomson $(1995 \mathrm{a}, 1997 \mathrm{a})$ shows that these properties are generally incompatible with the properties we introduced above. However, he shows that these incompatibilities only occur when the change in the parameter is such that it turns an economy in which there is "too much" to divide into an economy in which there is "too little" to divide, or vice versa. We call a change where this does not occur one-sided: let $N, \bar{N} \in \mathcal{N}, e=(R, \Omega) \in \mathcal{E}^{N}$, and $\bar{e}=(\bar{R}, \bar{\Omega}) \in \mathcal{E}^{\bar{N}}$. If either $\left[\sum_{N} p\left(R_{i}\right) \geq \Omega\right.$ and $\left.\sum_{\bar{N}} p\left(\bar{R}_{i}\right) \geq \bar{\Omega}\right]$ or $\left[\sum_{N} p\left(R_{i}\right) \leq \Omega\right.$ and $\left.\sum_{\bar{N}} p\left(\bar{R}_{i}\right) \leq \bar{\Omega}\right]$, then $\bar{e}$ is a one-sided change of $e$.

Thomson shows that a wide class of rules satisfies one or even several "one-sided" versions of the solidarity properties, i.e., solidarity is only required for one-sided changes.

First, we consider arrivals of new agents such that the implied change in the initial economy is one-sided, keeping the preferences of the remaining agents and the endowment fixed (Thomson, 1995a). One-sided population-monotonicity states that either all agents initially present (weakly) lose or all (weakly) gain. ${ }^{3}$

Let $N, M \in \mathcal{N}, N \subseteq M$, and $R \in \mathcal{R}^{M}$. Then, the restriction $\left(R_{i}\right)_{i \in N} \in \mathcal{R}^{N}$ of $R$ to $N$ is denoted by $R_{N}$.

One-sided population-monotonicity: $\quad$ For all $N, \bar{N} \in \mathcal{N}$, all $e=(R, \Omega) \in \mathcal{E}^{N}$, and all $\bar{e}=(\bar{R}, \Omega) \in \mathcal{E}^{N}$, if $N \subseteq \bar{N}, R=\bar{R}_{N}$, and $\bar{e}$ is a one-sided change of $e$, then either [for all $\left.i \in N, \varphi_{i}(e) R_{i} \varphi_{i}(\bar{e})\right]$ or [for all $i \in N, \varphi_{i}(\bar{e}) R_{i} \varphi_{i}(e)$ ].

Next, we consider a one-sided change of one agent's preference relation, keeping the preferences of the remaining agents, the set of agents, and the endowment fixed (Thomson, 1997a). Welfare-domination under preference-replacement, or replacement-domination for short, states that either all remaining agents (weakly) lose or all (weakly) gain. ${ }^{4}$

For $N, M \in \mathcal{N}$ with $N \subseteq M$ let $M \backslash N:=\{i \in M \mid i \notin N\}$. Let $N \in \mathcal{N}, R, \bar{R} \in \mathcal{R}^{N}$, and $j \in N$. If $R_{N \backslash\{j\}}=\bar{R}_{N \backslash\{j\}}$ and $R_{j} \neq \bar{R}_{j}$, then we call $\bar{R}$ a $j$-deviation from $R$.

One-sided replacement-domination: For all $N \in \mathcal{N}$, all $e=(R, \Omega), \bar{e}=(\bar{R}, \Omega) \in \mathcal{E}^{N}$, and all $j \in N$, if $\bar{R}$ is a $j$-deviation from $R$ and $\bar{e}$ is a one-sided change of $e$, then either [for all $\left.i \in N \backslash\{j\}, \varphi_{i}(e) R_{i} \varphi_{i}(\bar{e})\right]$ or [for all $\left.i \in N \backslash\{j\}, \varphi_{i}(\bar{e}) R_{i} \varphi_{i}(e)\right]$.

As a last property we introduce replication-invariance (Thomson, 1995a,1997a). Replication-invariance states that if an economy is replicated, i.e., the amount to divide and the preference profile are replicated, then the replica of the allocation assigned by the rule for the initial economy equals the allocation assigned by the rule for the replicated economy.

\footnotetext{
${ }^{3}$ For a survey on population-monotonicity we refer to Thomson (1995b).

${ }^{4}$ Replacement-domination has been studied in a variety of settings and we refer the interested reader to a recent review of the literature by Thomson (1999).
} 
Since replication-invariance is a well-known property and its formal description is somewhat cumbersome, for a formal statement we refer to Thomson (1995a,1997a). We call an economy $\bar{e}$, obtained by replication of an economy $e$, a replica of $e$. Similarly, we call an allocation $\bar{x}$ obtained by replication of an allocation $x$, a replica of $x$.

Replication-invariance: For all $N, \bar{N} \in \mathcal{N}$ and all $e=(R, \Omega) \in \mathcal{E}^{N}, \bar{e}=(\bar{R}, \bar{\Omega}) \in \mathcal{E}^{\bar{N}}$, if $\bar{e}$ is a replica of $e$, then $\varphi(\bar{e})$ is a replica of $\varphi(e)$.

\section{Replication-Invariance and the Uniform Rule}

In this section we first introduce the uniform rule and the characterizations of this rule that lead to the question whether replication-invariance in these characterizations is independent from the other properties.

\section{The uniform rule}

The following rule, known as the uniform rule, has played a central role in the literature of fair division when preferences are single-peaked.

Uniform rule $\boldsymbol{U}:$ For all $N \in \mathcal{N}$, all $e=(R, \Omega) \in \mathcal{E}^{N}$, and all $j \in N$,

$$
U_{j}(e):= \begin{cases}\min \left\{p\left(R_{j}\right), \lambda\right\} & \text { if } \sum_{N} p\left(R_{i}\right) \geq \Omega \\ \max \left\{p\left(R_{j}\right), \lambda\right\} & \text { if } \sum_{N} p\left(R_{i}\right) \leq \Omega\end{cases}
$$

where $\lambda$ solves $\sum_{N} U_{i}(e)=\Omega$.

So, in case of excess demand, i.e., $\sum_{N} p\left(R_{i}\right) \geq \Omega$, each agent either receives his peak amount or his allotment is greater than or equal to the allotment of each other agent. Similarly, in case of excess supply, i.e., $\sum_{N} p\left(R_{i}\right) \leq \Omega$, each agent either receives his peak amount or his allotment is smaller than or equal to the allotment of each other agent.

Another interpretation of this rule is the following "Walrasian interpretation". All agents are asked to maximize their preferences subject to a common upper or lower "budget bound" that is chosen such that feasibility is obtained for this list of maximizers. ${ }^{5}$

\section{Two characterizations of the uniform rule and two questions}

Thomson establishes the following characterization of the uniform rule (Thomson, 1995a, Theorem 4).

Theorem 1 (Thomson, 1995a). On the domain $\bigcup_{N \in \mathcal{N}} \mathcal{E}_{b}^{N}$, the uniform rule is the only rule that satisfies Pareto optimality, no-envy, one-sided population-monotonicity, and replication-invariance.

Question 1: Following the proof of this Theorem, Thomson asks whether replicationinvariance is independent from the other characterizing properties (see Thomson, 1995a,

\footnotetext{
${ }^{5}$ I wish to thank William Thomson for bringing this "Walrasian interpretation" of the uniform rule to my attention.
} 
page 242$){ }^{6}$

The next characterization of the uniform rule is due to Thomson (1997a, Theorem 1).

Theorem 2 (Thomson, 1997a).

On the domain $\bigcup_{N \in \mathcal{N}} \mathcal{E}^{N}$, the uniform rule is the only rule that satisfies Pareto optimality, no-envy, one-sided replacement-domination, and replication-invariance.

Question 2: Following the proof of this Theorem, Thomson asks whether replicationinvariance is independent from the other characterizing properties (see Thomson, 1997a, page 161).

We give the answers to Questions 1 and 2 by means of a single rule, unequal to the uniform rule, that satisfies Pareto optimality, no-envy, one-sided population-monotonicity, one-sided replacement-domination, but not replication-invariance.

Before we define the "absorbing agent" rule, we introduce some notation. Let $N \in \mathcal{N}$. Then, by $\mathcal{A}^{N} \subsetneq \mathcal{E}^{N}$ we denote the following subclass of economies: for $e=(R, \Omega) \in \mathcal{A}^{N}$ we have that (i) there is too much to distribute, i.e., $z(e)<0$ and (ii) there is exactly one agent, namely agent $j \in N$, with a relatively large peak amount $p\left(R_{j}\right)>\frac{\Omega}{2}$ (see Figure 1). For such an economy $e \in \mathcal{A}^{N}$, we construct the allocation assigned at $e$ as follows.

Figure 1: An economy $e \in \mathcal{A}^{N}$.

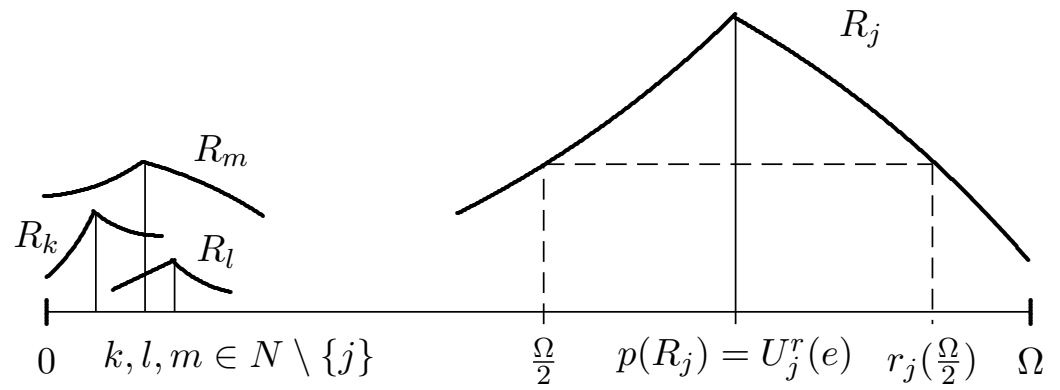

We start from the uniform allocation $U(e)$ and let agent $j$ "absorb" some part of the excess supply the remaining agents, $i \in N \backslash\{j\}$, experience at their uniform allotments, denoted by the list $\left(U_{i}(e)\right)_{i \in N \backslash\{j\}}$. We denote the excess supply of the agents in $N \backslash\{j\}$ at the allocation $U(e)$ by $s_{j}(e)=\sum_{N \backslash\{j\}}\left(U_{i}(e)-p\left(R_{i}\right)\right)$.

Note that in order to preserve same-sidedness, we let agent $j$ absorb at most $s_{j}(e)$. However, when we let agent $j$ absorb part, or even the whole, of the excess supply $s_{j}(e)$, we do not want him to envy the other agents. Therefore, we introduce the sufficiently small upper bound $r_{j}\left(\frac{\Omega}{2}\right)$ for agent $j$ 's allotment. Recall that either $r_{j}\left(\frac{\Omega}{2}\right) I_{j} \frac{\Omega}{2}$ or $r_{j}\left(\frac{\Omega}{2}\right)=\infty$. Note that the allotments of all other agents are smaller than $\frac{\Omega}{2}$ and therefore, as long as agent $j$ 's allotment is between his peak amount $p\left(R_{j}\right)$ and $r_{j}\left(\frac{\Omega}{2}\right)$, he does not envy the other agents.

\footnotetext{
${ }^{6}$ Thomson also asks the question whether the uniform rule is the only rule that satisfies Pareto optimality, individual rationality from equal division, one-sided population-monotonicity, and replication-invariance.

The answer is that there is a large class of rules satisfying the properties mentioned in the question. Examples are the "proportional reallocation rule" $\operatorname{Pr}^{r}$ operated from equal division and the "maximally satiating reallocation rule" operated from equal division (Klaus, 1998; Klaus, Peters, and Storcken, 1998).
} 
Summarizing, if $N \in \mathcal{N}$ and $e=(R, \Omega) \in \mathcal{A}^{N}$, then we obtain the allocation assigned by the absorbing agent rule from $U(e)$ by letting agent $j$ absorb the amount $a(e):=\min \left\{s_{j}(e), r_{j}\left(\frac{\Omega}{2}\right)-p\left(R_{j}\right)\right\}$ and subtracting this amount as equally as possible (with the agents' peak amount as lower bounds) from the uniform allotments of the other agents (see Figures 2 and 3). We denote this allocation by $\varphi^{a}(e)$. If $N \in \mathcal{N}$ and $e=(R, \Omega) \notin \mathcal{A}^{N}$, then we apply the uniform rule.

Figure 2: The rule $\varphi^{a}$ for an economy $e \in \mathcal{A}^{N}$ with $a(e)=s_{j}(e)$.
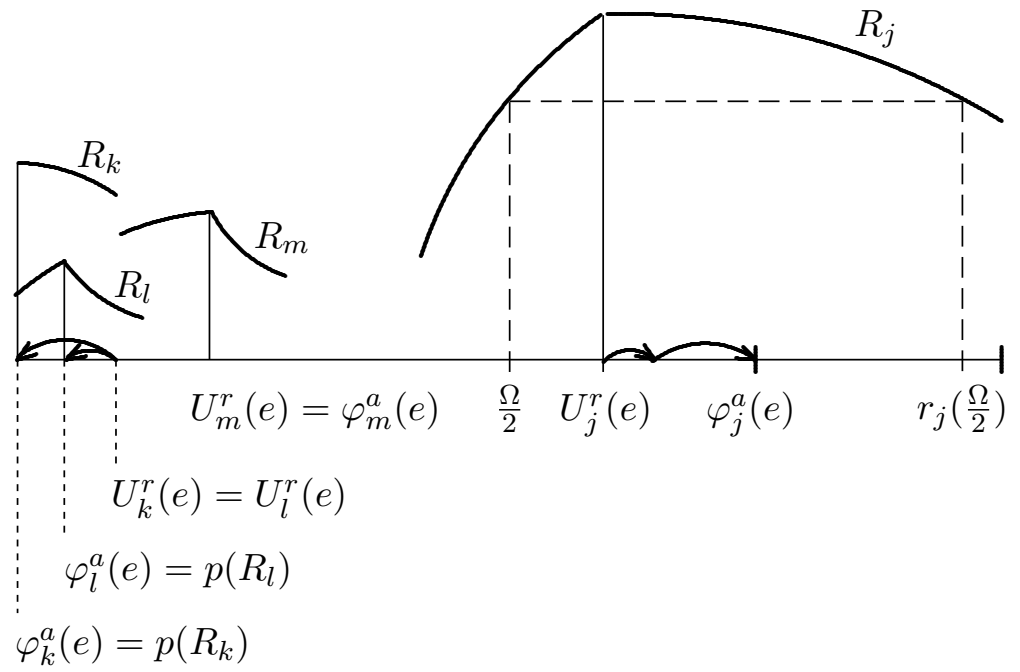

The absorbing agent rule $\varphi^{a}$ : For all $N \in \mathcal{N}$ and all $e=(R, \Omega) \in \mathcal{A}^{N}$, let $j \in N$ be such that $p\left(R_{j}\right)>\frac{\Omega}{2} .{ }^{7}$ Then, for all $i \in N$,

$$
\varphi_{i}^{a}(e)= \begin{cases}p\left(R_{j}\right)+a(e) & \text { if } i=j, \\ U_{i}\left(R_{N \backslash\{j\}}, \Omega-\varphi_{j}^{a}(e)\right) & \text { if } i \in N \backslash\{j\} .\end{cases}
$$

For all $N \in \mathcal{N}$ and all $e=(R, \Omega) \in \mathcal{E}^{N} \backslash \mathcal{A}^{N}$,

$$
\varphi^{a}(e)=U(e) .
$$

The rule $\varphi^{a}$ is by construction same-sided and therefore Pareto optimal. Since, we either assign the uniform allocation to an economy or change the uniform allocation in such a way that envy cannot arise, $\varphi^{a}$ satisfies no-envy. ${ }^{8}$

The following remark implies that the rule $\varphi^{a}$ does not satisfy replication-invariance.

Remark 1. Note that for all $N \in \mathcal{N}$, all $e=(R, \Omega) \in \mathcal{E}^{N}$, all $k \in \mathbb{N}, k \geq 2$, and all $k$-replica $\bar{e}=(\bar{R}, \bar{\Omega}) \in \mathcal{E}^{\bar{N}}$ of $e$, if $e \in \mathcal{A}^{N}$, then $\bar{e} \notin \mathcal{A}^{\bar{N}}$ and $\varphi^{a}(\bar{e})$ is not a $k$-replica of $\varphi^{a}(e)$.

Finally, by the following lemmas, we complete the proof that replication-invariance in Theorems 1 and 2 is independent from the other properties.

\footnotetext{
${ }^{7}$ Recall that the absorbing agent $j$ is unique and that $a(e):=\min \left\{s_{j}(e), r_{j}\left(\frac{\Omega}{2}\right)-p\left(R_{j}\right)\right\}$.

${ }^{8}$ The absorbing agent rule $\varphi^{a}$ satisfies other well-known properties, such as anonymity and individual rationality from equal division. It follows easily that $\varphi^{a}$ satisfies none of the properties strategy-proofness, peak-onliness, one-sided resource-monotonicity, consistency, and converse consistency. For a more detailed discussion, we refer to Klaus (1998).
} 
Figure 3: The rule $\varphi^{a}$ for an economy $e \in \mathcal{A}^{N}$ with $a(e)=r_{j}\left(\frac{\Omega}{2}\right)-p\left(R_{j}\right)$.

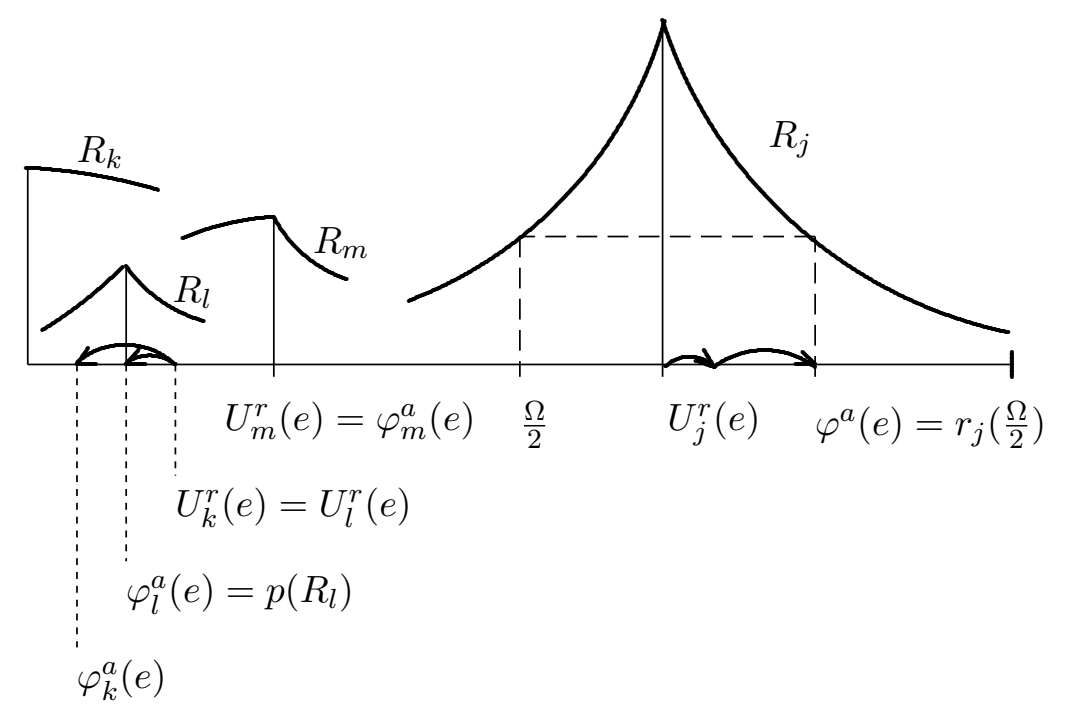

Lemma 1. The rule $\varphi^{a}$ satisfies one-sided population-monotonicity.

Lemma 2. The rule $\varphi^{a}$ satisfies one-sided replacement-domination.

Before we prove Lemmas 1 and 2, we introduce the solidarity property one-sided resource-monotonicity (Thomson, 1994). One-sided resource-monotonicity states that after a one-sided change of an economy that is induced by a change in the social endowment, either all agents (weakly) lose or all (weakly) gain.

One-sided resource-monotonicity: For all $N \in \mathcal{N}$ and all $e=(R, \Omega), \bar{e}=(R, \bar{\Omega}) \in \mathcal{E}^{N}$, if $\bar{e}$ is a one-sided change of $e$, then either [for all $i \in N, \varphi_{i}(e) R_{i} \varphi_{i}(\bar{e})$ ] or [for all $i \in N$, $\left.\varphi_{i}(\bar{e}) R_{i} \varphi_{i}(e)\right]$.

The uniform rule satisfies one-sided resource-monotonicity (Thomson, 1994).

Proof of Lemma 1. Let $N, \bar{N} \in \mathcal{N}, e=(R, \Omega) \in \mathcal{E}^{N}$, and $\bar{e}=(\bar{R}, \Omega) \in \mathcal{E}^{\bar{N}}$ be such that $N \subseteq \bar{N}$ and $R=\bar{R}_{N}$. Now, it is sufficient to show that, if $\bar{e}$ is a one-sided change of $e$, then either (i) [for all $i \in N, \varphi_{i}^{a}(e) R_{i} \varphi_{i}^{a}(\bar{e})$ ] or (ii) [for all $\left.i \in N, \varphi_{i}^{a}(\bar{e}) R_{i} \varphi_{i}^{a}(e)\right]$. Let $\bar{e}$ be a one-sided change of $e$.

Case 1: $\quad e \notin \mathcal{A}^{N}, \bar{e} \notin \mathcal{A}^{\bar{N}}$. Then, $\varphi^{a}(e)=U(e)$ and $\varphi^{a}(\bar{e})=U(\bar{e})$. Since, the uniform rule is one-sided population-monotonic, either (i) or (ii) holds.

Case 2: $\quad e \in \mathcal{A}^{N}, \bar{e} \notin \mathcal{A}^{\bar{N}}$. Hence, $z(\bar{e}) \leq 0, z(e)<0$, and there exists $j \in N$ such that $p\left(R_{j}\right)>\frac{\Omega}{2}$. Since $\bar{e} \notin \mathcal{A}^{\bar{N}}$, we have $z(\bar{e})=0$. So, by Pareto optimality, for all $i \in N$, $\varphi_{i}^{a}(\bar{e})=p\left(R_{i}\right)$. This implies (ii).

Case 3: $e \notin \mathcal{A}^{N}, \bar{e} \in \mathcal{A}^{\bar{N}}$. Hence, $z(e) \leq z(\bar{e})<0$ and there exists $j \in \bar{N} \backslash N$ such that $p\left(\bar{R}_{j}\right)>\frac{\Omega}{2}$. Let $R^{\prime}=\bar{R}_{\bar{N} \backslash\{j\}} \in \mathcal{R}^{\bar{N} \backslash\{j\}}$. Then, for all $i \in N$,

$$
\varphi_{i}^{a}(e)=U_{i}(e) \text { and } \varphi_{i}^{a}(\bar{e})=U_{i}\left(R^{\prime}, \Omega-\varphi_{j}^{a}(\bar{e})\right) .
$$


Since the uniform rule is one-sided resource-monotonic and $\left(R^{\prime}, \Omega\right),\left(R^{\prime}, \Omega-\varphi_{j}^{a}(\bar{e})\right) \in \mathcal{E}^{\bar{N} \backslash\{j\}}$ are such that $\sum_{\bar{N} \backslash\{j\}} p\left(R_{i}^{\prime}\right) \leq \Omega-\varphi_{j}^{a}(\bar{e})<\Omega$, it follows that for all $i \in N \subseteq \bar{N} \backslash\{j\}$,

$$
U_{i}\left(R^{\prime}, \Omega-\varphi_{j}^{a}(\bar{e})\right) R_{i} U_{i}\left(R^{\prime}, \Omega\right)
$$

Since the uniform rule is one-sided population-monotonic and $e=(R, \Omega) \in \mathcal{E}^{N},\left(R^{\prime}, \Omega\right) \in$ $\mathcal{E}^{\bar{N} \backslash\{j\}}$ are such that $N \subseteq \bar{N} \backslash\{j\}, R=R_{N}^{\prime}$, and $\sum_{\bar{N} \backslash\{j\}} p\left(R_{i}^{\prime}\right) \leq \Omega$, it follows that for all $i \in N$,

$$
U_{i}\left(R^{\prime}, \Omega\right) R_{i} U_{i}(e) .
$$

Thus, (1), (2), and (3) together imply that for all $i \in N, \varphi_{i}^{a}(\bar{e}) R_{i} \varphi_{i}^{a}(e)$. Hence, (ii) holds.

Case 4: $e \in \mathcal{A}^{N}, \bar{e} \in \mathcal{A}^{\bar{N}}$. Hence, $z(e) \leq z(\bar{e})<0$ and there exists $j \in N \subseteq \bar{N}$ such that $p\left(R_{j}\right)>\frac{\Omega}{2}$. Let $R^{\prime}=R_{N \backslash\{j\}} \in \mathcal{R}^{N \backslash\{j\}}$ and $\bar{R}^{\prime}=\bar{R}_{\bar{N} \backslash\{j\}} \in \mathcal{R}^{\bar{N} \backslash\{j\}}$. Then,

$$
\begin{aligned}
\varphi_{i}^{a}(e) & = \begin{cases}p\left(R_{j}\right)+a(e) & \text { if } i=j, \\
U_{i}\left(R^{\prime}, \Omega-\varphi_{j}^{a}(e)\right) & \text { if } i \in N \backslash\{j\},\end{cases} \\
\varphi_{i}^{a}(\bar{e}) & = \begin{cases}p\left(R_{j}\right)+a(\bar{e}) & \text { if } i=j, \\
U_{i}\left(\bar{R}^{\prime}, \Omega-\varphi_{j}^{a}(\bar{e})\right) & \text { if } i \in \bar{N} \backslash\{j\} .\end{cases}
\end{aligned}
$$

Note that $U_{j}(e)=p\left(R_{j}\right)=U_{j}(\bar{e})$. Therefore, $\Omega-U_{j}(e)=\sum_{N \backslash\{j\}} U_{i}(e)=\sum_{\bar{N} \backslash\{j\}} U_{i}(\bar{e})$ and $s_{j}(e) \geq s_{j}(\bar{e})$. Hence, $a(e) \geq a(\bar{e})$ and $p\left(R_{j}\right) \leq \varphi_{j}^{a}(\bar{e}) \leq \varphi_{j}^{a}(e)$. Thus,

$$
\varphi_{j}^{a}(\bar{e}) R_{j} \varphi_{j}^{a}(e)
$$

Now, we have either

(a) $a(\bar{e})=s_{j}(\bar{e})$ or

(b) $a(\bar{e})=r_{j}\left(\frac{\Omega}{2}\right)-p\left(R_{j}\right)$.

Suppose (a) holds. Then, for all $i \in N \backslash\{j\}, \varphi_{i}^{a}(\bar{e})=p\left(R_{i}\right)$. Thus, for all $i \in N \backslash\{j\}$,

$$
\varphi_{i}^{a}(\bar{e}) R_{i} \varphi_{i}^{a}(e)
$$

Hence, (5) and (6) imply (ii).

Suppose (b) holds. Then, $a(\bar{e})=a(e)$ and $\Omega-\varphi_{j}^{a}(\bar{e})=\Omega-\varphi_{j}^{a}(e)$. Since the uniform rule is one-sided population-monotonic and $\left(R^{\prime}, \Omega-\varphi_{j}^{a}(e)\right) \in \mathcal{E}^{N \backslash\{j\}},\left(\bar{R}^{\prime}, \Omega-\varphi_{j}^{a}(\bar{e})\right) \in \mathcal{E}^{\bar{N} \backslash\{j\}}$ are such that $N \backslash\{j\} \subseteq \bar{N} \backslash\{j\}, R^{\prime}=\bar{R}_{N \backslash\{j\}}^{\prime}$, and $\sum_{\bar{N} \backslash\{j\}} p\left(\bar{R}_{i}\right) \leq \Omega-\varphi_{j}^{a}(e)$, it follows that for all $i \in N \backslash\{j\}$,

$$
U_{i}\left(\bar{R}^{\prime}, \Omega-\varphi_{j}^{a}(\bar{e})\right) R_{i} U_{i}\left(R^{\prime}, \Omega-\varphi_{j}^{a}(e)\right) .
$$

Thus, by (4), for all $i \in N \backslash\{j\}$,

$$
\varphi_{i}^{a}(\bar{e}) R_{i} \varphi_{i}^{a}(e)
$$

Hence, (5) and (8) imply (ii).

Proof of Lemma 2. Let $N \in \mathcal{N}, e=(R, \Omega) \in \mathcal{E}^{N}, \bar{e}=(\bar{R}, \Omega) \in \mathcal{E}^{N}$, and $k \in N$ be such that $\bar{R}$ is a $k$-deviation from $R$. It is sufficient to show that if $\bar{e}$ is a one-sided change of $e$, then either (i) [for all $i \in N \backslash\{k\}, \varphi_{i}(e) R_{i} \varphi_{i}(\bar{e})$ ] or (ii) [for all $i \in N \backslash\{k\}, \varphi_{i}^{a}(\bar{e}) R_{i} \varphi_{i}^{a}(e)$ ]. Let $\bar{e}$ be a one-sided change of $e$. 
Case 1: $\quad e \notin \mathcal{A}^{N}, \bar{e} \notin \mathcal{A}^{N}$. Then, $\varphi^{a}(e)=U(e)$ and $\varphi^{a}(\bar{e})=U(\bar{e})$. Since, the uniform rule satisfies one-sided replacement-domination, either (i) or (ii) holds.

Case 2: $\quad e \notin \mathcal{A}^{N}, \bar{e} \in \mathcal{A}^{N}$. Hence, $\bar{R}$ and $R$ are such that $p\left(R_{k}\right) \leq \frac{\Omega}{2}, p\left(\bar{R}_{k}\right)>\frac{\Omega}{2}$, and $z(e) \leq z(\bar{e})<0$. Thus, $U_{k}(e) \leq \frac{\Omega}{2}<p\left(\bar{R}_{k}\right) \leq \varphi_{k}^{a}(\bar{R}, \Omega)$. Let $R^{\prime}=R_{N \backslash\{k\}} \in \mathcal{R}^{N \backslash\{k\}}$. Then, for all $i \in N \backslash\{k\}$,

$$
\varphi_{i}^{a}(e)=U_{i}(e)=U_{i}\left(R^{\prime}, \Omega-U_{k}(e)\right) \text { and } \varphi_{i}^{a}(\bar{e})=U_{i}\left(R^{\prime}, \Omega-\varphi_{k}^{a}(\bar{e})\right) .
$$

Since the uniform rule is one-sided resource-monotonic and $\left(R^{\prime}, \Omega-U_{k}(e)\right),\left(R^{\prime}, \Omega-\varphi_{k}^{a}(\bar{e})\right) \in$ $\mathcal{E}^{N \backslash\{k\}}$ are such that $\sum_{N \backslash\{k\}} p\left(R_{i}\right) \leq \Omega-\varphi_{k}^{a}(\bar{e}) \leq \Omega-U_{k}(e)$, it follows that for all $i \in N \backslash\{k\}$,

$$
U_{i}\left(R^{\prime}, \Omega-\varphi_{k}^{a}(\bar{e})\right) R_{i} U_{i}\left(R^{\prime}, \Omega-U_{k}(e)\right) .
$$

Hence, (9) and (10) imply (ii).

Case 3: $\quad e \in \mathcal{A}^{N}, \bar{e} \notin \mathcal{A}^{N}$. Similar to Case 2 by interchanging the roles of $\bar{R}$ and $R$.

Case 4: $e \in \mathcal{A}^{N}, \bar{e} \in \mathcal{A}^{N}$. Without loss of generality we assume that $\bar{R}$ is a $k$-deviation from $R$ such that $p\left(\bar{R}_{k}\right) \geq p\left(R_{k}\right)$. Hence, $z(e) \leq z(\bar{e})<0$ and there exists $j \in N$ such that $p\left(R_{j}\right)>\frac{\Omega}{2}$ and $p\left(\bar{R}_{j}\right)>\frac{\Omega}{2}$. Let $R^{\prime}=R_{N \backslash\{j\}} \in \mathcal{R}^{N \backslash\{j\}}$ and $\bar{R}^{\prime}=\bar{R}_{N \backslash\{j\}} \in \mathcal{R}^{N \backslash\{j\}}$. Then,

$$
\begin{aligned}
\varphi_{i}^{a}(e) & = \begin{cases}p\left(R_{j}\right)+a(e) & \text { if } i=j, \\
U_{i}\left(R^{\prime}, \Omega-\varphi_{j}^{a}(e)\right) & \text { if } i \in N \backslash\{j\},\end{cases} \\
\varphi_{i}^{a}(\bar{e}) & = \begin{cases}p\left(\bar{R}_{j}\right)+a(\bar{e}) & \text { if } i=j, \\
U_{i}\left(\bar{R}^{\prime}, \Omega-\varphi_{j}^{a}(\bar{e})\right) & \text { if } i \in N \backslash\{j\} .\end{cases}
\end{aligned}
$$

Case 4.1: $\quad k=j$. Since the uniform rule is one-sided resource-monotonic and $\left(R^{\prime}, \Omega-\varphi_{j}^{a}(e)\right)$, $\left(\bar{R}^{\prime}, \Omega-\varphi_{j}^{a}(\bar{e})\right) \in \mathcal{E}^{N \backslash\{j\}}$ are such that $R^{\prime}=\bar{R}^{\prime}$ and $z\left(R^{\prime}, \Omega-\varphi_{j}^{a}(e)\right) \leq 0$ and $z\left(\left(\bar{R}^{\prime}, \Omega-\varphi_{j}^{a}(\bar{e})\right) \leq\right.$ 0 , it follows that for all $i \in N \backslash\{j\}$, either (i) or (ii).

Case 4.2: $\quad k \neq j$. Since $p\left(\bar{R}_{k}\right) \geq p\left(R_{k}\right)$, it follows that $s_{j}(e) \geq s_{j}(\bar{e})$. Hence, $a(e) \geq a(\bar{e})$ and $\varphi_{j}^{a}(\bar{e}) \leq \varphi_{j}^{a}(e)$. Thus,

$$
\varphi_{j}^{a}(\bar{e}) R_{j} \varphi_{j}^{a}(e)
$$

Now, we have either

(a) $a(\bar{e})=s_{j}(\bar{e})$ or

(b) $a(\bar{e})=r_{j}\left(\frac{\Omega}{2}\right)-p\left(R_{j}\right)$.

Suppose (a) holds. Hence, for all $i \in N \backslash\{j\}, \varphi_{i}^{a}(\bar{e})=p\left(R_{i}\right)$. Thus, for all $i \in N \backslash\{j, k\}$,

$$
\varphi_{i}^{a}(\bar{e}) R_{i} \varphi_{i}^{a}(e) .
$$

Hence, (12) and (13) imply (ii).

Suppose (b) holds. Hence, $a(\bar{e})=a(e)$ and $\Omega-\varphi_{j}^{a}(\bar{e})=\Omega-\varphi_{j}^{a}(e)$. Since the uniform rule satisfies one-sided replacement-domination and $\left(R^{\prime}, \Omega-\varphi_{j}^{a}(e)\right),\left(\bar{R}^{\prime}, \Omega-\varphi_{j}^{a}(\bar{e})\right) \in \mathcal{E}^{N \backslash\{j\}}$, $k \in N$, are such that $p\left(\bar{R}_{k}\right) \geq p\left(R_{k}\right)$ and $R_{N^{\prime} \backslash\{k\}}^{\prime}=\bar{R}_{N^{\prime} \backslash\{k\}}^{\prime}$, it follows that for all $i \in$ $N^{\prime} \backslash\{k\}=N \backslash\{j, k\}$,

$$
U_{i}\left(\bar{R}^{\prime}, \Omega-\varphi_{j}^{a}(\bar{e})\right) R_{i} U_{i}\left(R^{\prime}, \Omega-\varphi_{j}^{a}(e)\right) .
$$

Thus, by (11) and (14), for all $i \in N \backslash\{j, k\}$,

$$
\varphi_{i}^{a}(\bar{e}) R_{i} \varphi_{i}^{a}(e)
$$

Hence, (12) and (15) imply (ii).

Now, Remark 1, together with Lemmas 1 and 2 establish the answers to Questions 1 and 2. 


\section{References}

Benassy JP (1982) The Economics of Market Equilibrium. Academic Press, San Diego

Klaus B (1998) Fair Allocation and Reallocation: An Axiomatic Study. Ph.D. Thesis, Maastricht University, The Netherlands, Unigraphic, Maastricht

Klaus B, Peters H, Storcken T (1998) Strategy-Proof Division with Single-Peaked Preferences and Individual Endowments. Social Choice and Welfare 15: 297-311

Sprumont Y (1991) The Division Problem with Single-Peaked Preferences: A characterization of the Uniform Allocation Rule. Econometrica 59: 509-519

Thomson W(1994) Resource-Monotonic Solutions to the Problem of Fair Division when Preferences are Single-Peaked. Social Choice and Welfare 11: 205-223

Thomson W (1995a) Population-Monotonic Solutions to the Problem of Fair Division when Preferences are Single-Peaked. Economic Theory 5: 229-246

Thomson W (1995b) Population monotonic allocation rules. In: Barnett WA, Moulin H, Salles M, Schofield N (eds.) Social choice, welfare and ethics. Cambridge University Press.

Thomson W (1997a) The Replacement Principle in Economies with Single-Peaked Preferences. Journal of Economic Theory 76: 145-168

Thomson W (1997b) The Theory of Fair Allocation. Princeton University Press, forthcoming

Thomson W (1999) Welfare-domination under preference-replacement: A survey and open questions. Social Choice and Welfare 16:373-394 